\title{
Schumann, Svantje
}

\section{Technische Ereignisse in Stummfilmen erschließen. Eine Untersuchung der Bildungsprozesse von Kindern}

Landwehr, Brunhild [Hrsg.]; Mammes, Ingelore [Hrsg.]; Murmann, Lydia [Hrsg.]: Technische Bildung im Sachunterricht der Grundschule. Elementar bildungsbedeutsam und dennoch vernachlässigt? Bad Heilbrunn : Verlag Julius Klinkhardt 2021, S. 95-116. - (Forschungen zur Didaktik des Sachunterrichts; 12)

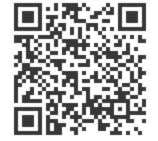

\section{Quellenangabe/ Reference:}

Schumann, Svantje: Technische Ereignisse in Stummfilmen erschließen. Eine Untersuchung der Bildungsprozesse von Kindern - In: Landwehr, Brunhild [Hrsg.]; Mammes, Ingelore [Hrsg.]; Murmann, Lydia [Hrsg.]: Technische Bildung im Sachunterricht der Grundschule. Elementar bildungsbedeutsam und dennoch vernachlässigt? Bad Heilbrunn : Verlag Julius Klinkhardt 2021, S. 95-116 - URN: urn:nbn:de:0111-pedocs-215340 - DOI: 10.25656/01:21534

https://nbn-resolving.org/urn:nbn:de:0111-pedocs-215340

https://doi.org/10.25656/01:21534

in Kooperation mit / in cooperation with:

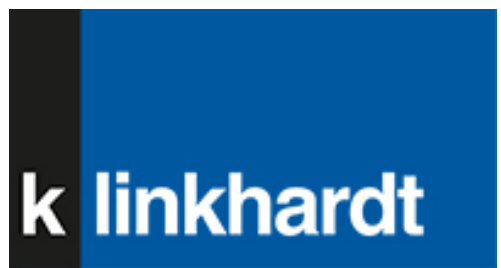

http://www.klinkhardt.de

\section{Nutzungsbedingungen}

Dieses Dokument steht unter folgender Creative Commons-Lizenz: http://creativecommons.org/licenses/by-nc-sa/4.0/deed.de - Sie dürfen das Werk bzw. den Inhalt unter folgenden Bedingungen vervielfältigen, verbreiten und öffentlich zugänglich machen sowie Abwandlungen und Bearbeitungen des Werkes bzw. Inhaltes anfertigen: Sie müssen den Namen des Autors/Rechteinhabers in der von ihm festgelegten Weise nennen. Dieses Werk bzw. der Inhalt darf nicht für kommerzielle Zwecke verwendet werden. Die neu entstandenen Werke bzw. Inhalte dürfen nur unter Verwendung von Lizenzbedingungen weitergegeben werden, die mit denen dieses Lizenzvertrages identisch oder vergleichbar sind.

Mit der Verwendung dieses Dokuments erkennen Sie die Nutzungsbedingungen an.

\section{Terms of use}

This document is published under following Creative Commons-License: http://creativecommons.org/licenses/by-nc-sa/4.0/deed.en - You may copy, distribute and transmit, adapt or exhibit the work in the public and alter, transform or change this work as long as you attribute the work in the manner specified by the author or licensor. You are not allowed to make commercial use of the work. If you alter, transform, or change this work in any way, you may distribute the resulting work only under this or a comparable license.

By using this particular document, you accept the above-stated conditions of use.

\section{Kontakt / Contact:}

\section{peDOCs}

DIPF | Leibniz-Institut für Bildungsforschung und Bildungsinformation Informationszentrum (IZ) Bildung

E-Mail: pedocs@dipf.de

Internet: www.pedocs.de

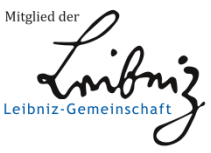


Forschungen zur Didaktik des Sachunterrichts

Brunhild Landwehr Ingelore Mammes

Lydia Murmann

(Hrsg.)

Technische Bildung

im Sachunterricht der Grundschule

Elementar bildungsbedeutsam und dennoch vernachlässigt? 
Forschungen zur Didaktik des Sachunterrichts

Band 12 


\section{Brunhild Landwehr Ingelore Mammes Lydia Murmann \\ (Hrsg.)}

\section{Technische Bildung im Sachunterricht der Grundschule}

Elementar bildungsbedeutsam und dennoch vernachlässigt? 
Schriftenreihe der

Gesellschaft für Didaktik des Sachunterrichts e.V.

Die Gesellschaft für Didaktik des Sachunterrichts (GDSU) e.V. ist ein Zusammenschluss von Lehrenden aus Hochschule, Lehrerfortbildung, Lehrerweiterbildung und Schule. Ihre Aufgabe ist die Förderung der Didaktik des Sachunterrichts als wissenschaftliche Disziplin in Forschung und Lehre sowie die Vertretung der Belange des Schulfaches Sachunterricht. www.gdsu.de

Dieser Titel wurde in das Programm des Verlages mittels eines Peer-Review-Verfahrens aufgenommen. Für weitere Informationen siehe www.klinkhardt.de.

Bibliografische Information der Deutschen Nationalbibliothek Die Deutsche Nationalbibliothek verzeichnet diese Publikation in der Deutschen Nationalbibliografie; detaillierte bibliografische Daten sind im Internet abrufbar über http://dnb.d-nb.de.

2021.n. () by Julius Klinkhardt.

Druck und Bindung: AZ Druck und Datentechnik, Kempten.

Printed in Germany 2021.

Gedruckt auf chlorfrei gebleichtem alterungsbeständigem Papier.

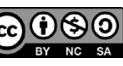

Die Publikation (mit Ausnahme aller Fotos, Grafiken und Abbildungen) ist veröffent-

licht unter der Creative Commons-Lizenz: CC BY-NC-SA 4.0 International

https://creativecommons.org/licenses/by-nc-sa/4.0/

ISBN 978-3-7815-5869-4 digital

doi.org/10.35468/5869

ISBN 978-3-7815-2430-9 print 


\section{Inhaltsverzeichnis}

Brunhild Landwehr, Ingelore Mammes und Lydia Murmann

Editorial

Andreas Schmitt und Tanja Fellensiek

„Windräder werden mit Strom betrieben, um Wind zu erzeugen ...

oder umgekehrt!?" - Schülervorstellungen und Konzeptwechsel zum

Thema Windenergie im Sachunterricht

Stefan Fletcher und Anja Kleinteich

Vorstellungen von Grundschüler*innen zum Ende der Primarstufe über den grundsätzlichen Aufbau eines komplexen technischen Systems zur Energieerzeugung untersucht am Beispiel der Konstruktion eines Wasserkraftwerks aus vorgegebenen Teilsystemen

Swantje Dölle

LERNnetze - Lernunterstützung im technischen Sachunterricht

Erprobung kognitiv aktivierender und inhaltlich strukturierender

Maßnahmen der Lernunterstützung und

Überprüfung der Angebotsnutzung

\section{Victoria Adenstedt}

Attributionen von Grundschulkindern zur Erklärung von

Leistungsergebnissen bei technischen Alltagsaufgaben

\section{Svantje Schumann}

Technische Ereignisse in Stummfilmen erschließen -

eine Untersuchung der Bildungsprozesse von Kindern

Lennart Goecke, Jurik Stiller und Julia Schwanewedel

Algorithmusverständnis in der Primarstufe - Eine Studie im Kontext

des Einsatzes von programmierbarem Material

Sabine Martschinke, Susanne Palmer Parreira und Ralf Romeike

Informatische (Grund-)Bildung schon in der Primarstufe?

Erste Ergebnisse aus einer Evaluationsstudie 
6| Inhaltsverzeichnis

Eva Gläser und Christina Krumbacher

Ausstattung zur technischen Bildung mangelhaft?

Eine quantitative Studie zur Situation an Grundschulen

Verzeichnis der Autorinnen und Autoren ............................................... 167 


\section{Technische Ereignisse in Stummfilmen erschließen - eine Untersuchung der Bildungsprozesse von Kindern}

\section{Skizzierung des Forschungsprojekts und der Fragestellung}

Technische Bildungsinhalte werden als unverzichtbare Elemente einer zeitgemäßen Allgemeinbildung und elementaren Grundbildung angesehen (Sachs 2001, Kruse \& Labudde 2016). Nach wie vor findet aber nur wenig Technikunterricht im Rahmen des Sachunterrichts statt (vgl. Linke 2013). Hempel (2007) plädiert vor dem Hintergrund der Annahme, dass die Förderung von technischem Verständnis als Voraussetzung eine Diagnose von kindlicher Lebenswelt und kindlichen Bildungsprozessen benötigt, für die verstärkte Beforschung von Bildungsprozessen. Hingewiesen wird darauf, dass kaum erforscht ist, wie Bildungsprozesse im Technikbereich verlaufen und wie Kinder zu Interpretationen über Technik kommen (Kruse \& Labudde 2016). Vor diesem Hintergrund wird im Folgenden eine Forschungsstudie vorgestellt, die auf Erkenntnisgewinn zu technikbezogenen Bildungsprozessen im Sachunterricht abzielt. Es wurden Technik-Stummfilme entwickelt, die anschließend von Kindern der Primarstufe in Partnerarbeit an Notebook-Stationen angeschaut wurden. Im Gespräch miteinander versuchten sie, sich die Filme zu erschließen.

Erschließen ist zu einem zentralen Begriff der Sachunterrichtsdidaktik geworden (vgl. GDSU 2013, 9). Kahlert (2009, 17) sieht im Erschließungsbegriff das vielleicht am meisten Einvernehmlichkeit für sich in Anspruch nehmen könnende Leitbild des Sachunterrichts. Gleichzeitig fällt der Mangel an Wissen über Erschließungsprozesse bei Kindern auf. Es besteht die Forderung, Rekonstruktionsleistungen der Kinder stärker als Ausgangspunkte von Erschließungsprozessen zu nutzen (vgl. Royar \& Streit 2010) und unmittelbarer an kindlichen Interessen anzuknüpfen (Schönknecht \& Maier 2012). In Bezug auf Erschließungsprozesse im Sachunterricht wird davon ausgegangen, dass vor allem solche Bildungsprozesse nachhaltig auf Kinder im Primarschulalter wirken, bei denen die Kinder sich Erklärungen selbst konstruieren können (vgl. u. a. Wagenschein 2005, Bielmeier 2012).

Bezüglich des oben beschriebenen Forschungssettings - Stummfilm-Erschließung durch Kinder - wurden nun zwei gegenläufige Annahmen aufgestellt: Erstens: 
Stummfilme regen dazu an, genau zu beobachten und Beobachtungen sprachlich auszudrücken bzw. Fragen zu formulieren - so werden abduktive und induktive Erschließungsprozesse potentiell unterstützt (vgl. Geiss \& Schumann 2014), denn Sprache ist das Medium des Austauschs über Sinngehalte und die Grundlage von Deutung (vgl. Leber \& Oevermann 1994, 384f.). Zweitens: Kinder im Primarschulalter benötigen zum abduktiven und induktiven Erschließen von technischen Ereignissen bzw. Gegenständen u. a. die originale Begegnung und die Möglichkeit des handelnden Erschließens (u. a. GDSU 2018) - beide letztgenannten Zugänge fehlen im Fall des Angebots „Stummfilme“ und erschweren abduktives und induktives Erschließen. Das Spannungsfeld, das von beiden Annahmen gebildet wird, führt zu dem Anliegen, Erschließen von Technik näher zu untersuchen und Widersprüche dadurch ggf. auflösen zu können sowie, wenn möglich, bildungswirksame Maßnahmen zur Unterstützung rekonstruierender Lernprozesse im Bereich Technik abzuleiten.

\section{Der sachunterrichtsdidaktische Theorierahmen}

Um die Ergebnisse der Forschungsstudie später im Kontext aktueller fachdidaktischer Literatur beleuchten zu können, soll im Folgenden der aktuelle Forschungsstand bezüglich der Bedeutung des Handelns und der Sprache für Bildungsprozesse umrissen werden.

\subsection{Annahmen bezüglich der Bedeutung des Handelns für Erschließungsprozesse im Sachunterricht generell}

Im Sachunterricht wird oft von der Prämisse ausgegangen, dass Kinder vor allem von der Handlungsorientierung, der durch Handeln erwerbbaren Lernerfahrung, profitieren (vgl. u. a. Dewey 1933; Donaldson 1991; Soostmeyer 1998; 2002, Giest 2004; Kaiser 2004; Heck u. a. 2009, 46). Handlungsorientiertes Lernen ist laut Einsiedler (2015) sowie Gervé und Mayer (2018) ein Grundprinzip des naturwissenschaftlichen Sachunterrichts. Laut Hartinger (1997) ist es besonders wichtig für den Aufbau von Interesse.

Gleichzeitig wird immer wieder betont, dass für den Handlungsbegriff eine lerntheoretische Begriffsfundierung bzw. Konzeptfundierung fehle und er unscharf und unterbestimmt sei. Kritisiert wird, dass als handlungsorientiert deklarierter Unterricht in der Realität häufig auf ein bloßes Tun hinauslaufe und Reflexionsphasen vernachlässigt würden (Kahlert 2009, 184). Handlungsorientierter Unterricht ist empirisch wenig untersucht (Franz u. a. 2018, 9ff.); es besteht beispielsweise wenig Kenntnis darüber, in welchen Alters- und Entwicklungsstufen Kinder ggf. in besonderer Weise auf welche Formen eines handlungsgeprägten Zugangs zu Phänomenen angewiesen sind. 


\subsection{Annahmen bezüglich der Bedeutung des Handelns für den Technikunterricht speziell}

Die Annahmen in Bezug auf das Handeln im Bereich der Technischen Bildung stellen sich sehr heterogen dar. Teilweise wird für handlungsorientierten, teilweise für gedanklich-analytischen Technikunterricht plädiert. Köhnlein (2012, 471) beispielsweise sieht in der Schulung des „produktiven und konstruktiven Denkens das übergreifende formale Ziel im Bereich der technischen Dimension“" und plädiert sowohl für "das mentale Spiel mit Vorstellungsbildern“ (Köhnlein 2012, 472) als auch für „das Untersuchen, Probieren, Planen, Konstruieren und Erfinden" (Köhnlein 2012, 471). Empfohlen wird von der GDSU letztlich das kombinierte Angebot von Handlungs- und Verstehensprozessen: „Erst die Verknüpfung von Handlungs- und Verstehensprozessen ermöglicht das Erfassen von technischen Gegenständen, Prozessen und Abläufen, die Übertragung des Erfassten auf weitere technische Bereiche sowie die kritische Auseinandersetzung mit Technik." (GDSU 2013, 44)

\subsection{Annahmen bezüglich der Bedeutung der Sprache im Sachunterricht generell}

Sprache wird in der Sachunterrichtsdidaktik meistens unter der Fragestellung betrachtet, wie sich Fachunterricht auf die Sprachentwicklung auswirkt. Angenommen wird in der Literatur vor allem die positive Wirkung naturwissenschaftsbezogener Bildungsanlässe und -inhalte auf insbesondere den Wortschatz (Tomcin \& Reiners 2009, Gottwald 2012/2016, Neugebauer \& Nodari 2017).

Aussagen zum Zusammenhang von Sprache und Verständnis finden sich vor allem in allgemeinerer Form. Beispielsweise geht Sprache gemäß Butler (2006) über ein reines Benennen hinaus und kann als handelnder Bildungsprozessmodus verstanden werden - Sprache "tut“ also etwas: sie führt zu Deutungen. Insgesamt sind aber Untersuchungen zur Bedeutung von Sprache für den Aufbau von Fachverständnis kaum zu finden. Es gibt die Annahme, dass bewusste Versprachlichungen von naturwissenschaftlichen Sachverhalten allmählich das Verständnis und damit die Erkenntnis erweitern (Goedhart 1999, Aeschlimann u. a. 2008, Ostergaard \& Hugo 2008, Archie 2016). Wagenschein (1923/1996) sieht in einer sorgfältigen Versprachlichung von naturwissenschaftlichen Sachverhalten das Potential, Beziehungen zwischen alten und neuen Begriffen zu sehen und so eine Transformation sowohl im Bereich der Sprache als auch im Bereich des Verständnisses herbeiführen zu können.

Nur vereinzelt finden sich Beobachtungen und Aussagen zu Bildungsprozessen, die im Zusammenhang mit der Fachsprache stehen. Wagenschein geht von der Annahme aus, dass sich zunächst die Muttersprache entwickelt und daraus dann in einem Prozess der Ausschärfung, Beschränkung und Eindeutigkeitsbestim- 
mung bruchlos die Fachsprache hervorgeht (Wagenschein 1976, 1923/1996). Sowohl Wagenschein als auch Merzyn (1998) und Apolin (2004) vertreten die Auffassung, dass eine zu frühe Einführung von Fachbegriffen und ein zu frühes Bestehen auf korrekter Fachsprache zur Unbeliebtheit naturwissenschaftlichen Unterrichts beitrüge.

\subsection{Annahmen bezüglich der Bedeutung der Sprache im Technikunterricht speziell}

Auch wenn er sich nicht explizit auf den Bereich der Technik bezieht, so lassen sich einige Hinweise bzw. Beobachtungen Wagenscheins unmittelbar auf den Technikbereich beziehen. Gemeint ist die Beobachtung, dass Kinder, wenn sie sensibilisiert wurden für den Aspekt einer Definition, „,bald einen gewissen sportlichen Eifer, sich an Schärfe und Knappheit der Definition zu übertreffen" an den Tag legten und dabei erfahren würden, dass „man zuletzt bei gewissen Grundbegriffen haltmachen muß“ und dass man ,sich sowohl vor zu engen als auch vor zu weiten Definitionen zu hüten hat" (Wagenschein 1923/1996, 6). Noch schwieriger als gute Definitionen aufzustellen sei es, sprachlich Beziehungen bzw. Zusammenhänge zwischen verschiedenen Teilen eines Gegenstands auszudrücken. Um Zusammenhänge zu verstehen, kann man nach Wagenschein entweder von der Logik der Funktionsweise ausgehen (z. B. beim Fernrohr überlegen, wie der Weg des Lichts durch das Fernrohr ist und diesen nachvollziehen) oder von der Logik der Herstellung (z. B. die Reihenfolge des Zusammenbaus von Einzelteilen zum Ganzen betrachten) (Wagenschein 1923/1996, 10).

Köhnlein $(2012,32)$ ist der Meinung, dass das Potential des dialogischen Charakters von Erschließungsprozessen von der Naturwissenschaftsdidaktik bisher zu wenig ausgeschöpft wird. Mikelskis-Seifert \& Gromadecki (2006) fordern, Gesprächsabläufe im Sachunterricht stärker zu untersuchen. Und Merzyn schreibt: „Sprache spielt für den naturwissenschaftlichen Unterricht eine vielfach unterschätzte, eminent wichtige Rolle" (Merzyn 1998, 11). Wenn Sprache, und sei es nur der Vermutung nach, so eine wichtige Rolle für Erschließungsprozesse besitzt, ist erklärungsbedürftig, warum der versprachlichenden, potentiell argumentativen Auseinandersetzung von Kindern im Rahmen von Erschließungsprozessen im Sachunterricht so wenig Bedeutung seitens der Forschung geschenkt wird. 


\section{Methodik}

\subsection{Datenerhebung und Analyse}

Vorgestellt wird eine hermeneutische Fallstudie, die im Rahmen eines PgB MINT-Bildungsprojekts in einer fünften Klasse mit 16 Schülerinnen und Schülern einer Primarschule in der Schweiz generiert wurde (vgl. https://www.fhnw. $\mathrm{ch} /$ de/die-fhnw/hochschulen/ht/mint-bildung).

Im Klassenzimmer wurden zehn Notebook-Stationen aufgestellt - an jeder Station war ein anderer Stummfilm auf einem Video-Player abrufbar: Dübel, Reißverschluss, Wagenheber, Handschwengelpumpe, Mähdrescher, Handbohrmaschine, Heißklebepistole, Kugelschreiber, Fahrradschaltung, Schleuderschneeketten.

Die Kinder arbeiteten in Zweiergruppen an den Notebook-Stationen. Hinter dem Modell der Partnerarbeit steckt die Idee der Externalisierung von Gedankengängen. Die Bildungsforschung steht oft vor dem Problem, dass Lernprozesse von Kindern als innerer Prozess, als Dialog mit sich selbst stattfinden, so dass man kein Protokoll davon generieren kann. Und die Analyse von Protokollen mit hohen Anteilen nonverbaler Sequenzen beinhaltet die Gefahr, zu spekulativ zu werden. Für die Beforschung kindlicher Bildungsprozesse bietet es sich an, die Externalisierung bereits in das Setting des Kommunikationsrahmens einzuschalten, so dass man den Prozess unmittelbar analytisch begleiten kann und später die sich entwickelnde, fortlaufende Rekonstruktion vor sich hat.

Der Auftrag war, sich zu zweit den Film an einer Station anzuschauen und zu versuchen, den Film zu verstehen und sich über den Film auszutauschen. Es wurde darauf hingewiesen, dass es möglich ist, den Film mehrmals anzusehen oder zwischendrin anzuhalten. Die Kinder durften - dies war freiwillig - zudem Fragen, Beobachtungen oder Skizzen notieren. Wenn die Kinder das Gefühl hatten, eine Station abgeschlossen zu haben, konnten sie zu zweit selbstständig die nächste freie Notebook-Station aufsuchen. Zusätzlich zu den Filmen standen den Kindern nur an vier Notebook-Stationen Materialien zur Verfügung (z. B. an Station „Dübel“ große Dübel mit passenden Schraubhaken und an der Station „Handbohrmaschine“ mehrere Handbohrmaschinen, Holz und ein Satz Bohrer). So gab es in Hinblick auf die spätere Analyse eine Vergleichsmöglichkeit zwischen Stationen mit und Stationen ohne zur Verfügung gestelltes Material. Die Kinder taten sich freiwillig zu Zweiergruppen zusammen. Die aufgezeichneten Gespräche der Kinder (s. u.) wurden objektiv-hermeneutisch analysiert.

Es wird davon ausgegangen, dass thesengenerierende Methoden wie die Objektive Hermeneutik gerade in Bereichen mit viel Erkenntnisgewinnungsbedarf viel Erkenntnisgenerierungs-Potential haben. Die Methode der Objektiven Hermeneutik ist im Gegensatz zu einer subsumierenden Vorgehensweise eine rekonstruktive Methode. Sie zielt darauf ab, die typischen, d.h. charakteristischen Strukturen zu erforschender Phänomene zu entschlüsseln und die „hinter den Erscheinungen 
operierenden objektiven Gesetzmäßigkeiten ans Licht zu bringen” (Oevermann 1996, 1). Die Methode versucht, Lesarten bezüglich möglicher Bedeutungsstrukturen zu formulieren und auf diese Weise Thesen zu generieren.

Optimale Grundlage für die Anwendung der Objektiven Hermeneutik sind gerätebasiert aufgezeichnete Protokolle - hierbei gehen keine Daten verloren und die Aufzeichnung ist weder subjektbehaftet noch ,intelligent“. Eine in der sozialwissenschaftlichen Forschung gebräuchliche Alternative, die „Beobachtung der Beobachtung" wird von der Objektiven Hermeneutik abgelehnt, weil schriftliche, von beobachtenden Personen verfasste und eben nicht gerätebasierte Protokolle immer eine Mischform aus Datenerhebung und -auswertung darstellen; Datenerhebung und -interpretation lassen sich nicht mehr trennen. Im Zentrum stand insgesamt die Beobachtung des realen, möglichst wenig durch Vorgaben überprägten Interaktionsgeschehens.

Die Kinder wurden an den Stationen gefilmt und die Gespräche der Kinder wurden mit Hilfe eines neu entwickelten Verfahrens audiografischer Unterrichtsbeobachtung aufgezeichnet. Jede Schülerin/jeder Schüler wurde mit einem USBStick-großen Aufnahmegerät ausgestattet, welches mit Hilfe eines Clips an der Kleidung befestigt wurde. Die Audiografierung mittels Stick sollte dafür sorgen, dass individuelle Äußerungen nicht verloren gehen. Alle 16 Aufnahmegeräte wurden mit einem Synchronisations-Tonsignal versehen, um die Tonspuren später auf eine gemeinsame Zeitschiene legen zu können. Die Tonaufnahmen wurden später mit einem Mehrspur-Sequenzer am Computer nachbearbeitet, synchronisiert und geschnitten. Die so bearbeiteten MP3-Aufnahmen wurden manuell transkribiert und mit Hilfe der Methode der Objektiven Hermeneutik analysiert. Die Details des Aufnahme- und Transkriptionsverfahrens können bei der Autorin nachgefragt werden, eine ausführliche Darstellung ist bislang noch nicht publiziert.

Mit Hilfe der Studie sollen Erkenntnisse darüber generiert werden, wie Kinder sich bei der Begegnung mit den Technik-Stummfilmen verhalten und bei der Erschließung vorgehen - auch, um damit eine bessere Grundlage zu erhalten, die ggf. das Ableiten von bildungswirksamen Maßnahmen zur Unterstützung rekonstruktiver Erschließungsprozesse im Technikunterricht der Primarstufe zulässt.

\subsection{Stummfilm-Setting}

An den Notebook-Stationen kamen auf verschiedene Weise generierte Stummfilme zum Einsatz, die jeweils spezifische Merkmale aufweisen (alle entwickelten Stummfilme sind auf einer Online-Plattform aufgeschaltet und stehen sowohl für die Aus- und Weiterbildung von Pädagog*innen als auch für den Einsatz in Schulen sowie für die weitere Forschung zur Verfügung, siehe Schumann 2018b). Allen eingesetzten Stummfilmen ist gemeinsam, dass sie in einer Gesamtlänge von ca. 2 bis 3 Minuten den technischen Ablauf eines Vorgangs bzw. einen Gegenstand 
zeigen. Mit Ausnahme der beiden realgefilmten Videos „Fahrradschaltung“ und „Handbohrmaschine“ waren die eingesetzten Filme Animationen. Die Filme „Schleuderschneeketten“ und „Reißverschluss“ bestanden aus sowohl animierten als auch real gefilmten Abschnitten.

Im Fall der „Animation“ werden Einzelbilder erstellt und so angezeigt, dass sich für den Betrachter ein bewegtes Bild ergibt. Eine Animation analysiert die in der Realität existierenden Bewegungsabläufe, muss sie aber nicht 1:1 umsetzen, sondern kann z. B. einzelne Details vergrößert darstellen oder das Timing verändern, um sehr schnell ablaufende Prozesse zu verlangsamen und damit besser wahrnehmbar zu machen. Innenansichten können zugänglich gemacht oder Innenund Außenansichten gleichzeitig dargestellt werden.

Das Filmformat „real Gefilmtes“ zeigt Vorgänge/Ereignisse von filmisch bzw. fotografisch abgebildeten realen Dingen. Es hat den Vorteil, den Gegenstand unverfälscht vor sich zu haben, ohne dass Abstraktionsleistungen vollbracht werden müssen. Es geht vor allem um das Vorführen einer Handlung oder eines technischen Ereignisses.

\section{Analyse}

Die Analyse der Gesprächssequenzen (die Analyse der Aufschriebe wird aus Darstellungsgründen nur gestreift) mittels Objektiver Hermeneutik kann im Folgenden nur exemplarisch dargestellt werden.

Die Methode der Objektiven Hermeneutik (Oevermann u. a. 1979, Oevermann 1996, Wernet 2006) versteht sich als rekonstruktionslogisches, thesengenerierendes Verfahren. Rekonstruktionslogische Analysen begründen sich gerade auf dem Gebiet der Grundlagenforschung damit, dass sie weitgehend ohne noch sehr spekulative, empirisch kaum überprüfte theoretische Vorannahmen auskommen. Geht man davon aus, dass die bisherige Theorielage noch ungenügend empirisch untersucht ist, scheint es angemessen zu sein, sich möglichst von ihr zu lösen.

Bei der Methode wird ein Protokoll nach allen Seiten hin erschöpfend analysiert - es besteht der Anspruch, aus relativ wenigen Daten die maximale Erkenntnis, in Form von Thesen, zu generieren. Bezüglich der Datenauswertung gilt, dass die Objektive Hermeneutik nicht eine Methode des Verstehens im Sinne eines Nachvollzuges subjektiver Dispositionen oder eine Methode des Sich-Einfühlens, sondern eine strikt analytische Methode der lückenlosen Erschließung und Rekonstruktion von objektiven Sinn- und Bedeutungsstrukturen ist (Oevermann 1996: 16). Im Zuge der Datenauswertung wird versucht, von einer konkreten Äußerung beginnend die objektive Bedeutungsstruktur zu rekonstruieren, d. h. die objektiv geltenden Sinnstrukturen werden intersubjektiv überprüfbar an konkreten, les-, hörund sichtbaren Ausdrucksgestalten zu entziffern versucht (Oevermann 1996: 1). 
Es wird im Fall der Objektiven Hermeneutik nicht von einer bestehenden Theorie ausgegangen und von ihr ausgehend Hypothesen abgeleitet, die anschließend geprüft werden, sondern bei der Analyse des Materials werden Hypothesen bzw. Aussagen generiert. Dahinter steckt auch die Auffassung, dass eine Theorie (oder auch ein theoretischer Begriff) erst in der rekonstruierenden Darstellung einer konkreten Sache seine Gültigkeit erweisen kann. Wenn man eine Sequenz in hinreichender Länge bruchlos rekonstruiert hat, so die Annahme, bildet sich die Fallstrukturgesetzlichkeit ab, aus der heraus eine je konkrete Lebenspraxis ihre Wahl bzw. ihre Entscheidungen wiedererkennbar getroffen hat.

Einige grundlegenden Prinzipien, die für das Verständnis der Datenauswertung mit der Methode der Objektiven Hermeneutik eine besondere Bedeutung haben, sind, in Anlehnung an Wernet (2006):

- Das Totalitätsprinzip: Das Totalitätsprinzip bedeutet eine vollständige Bearbeitung dessen, was textlich vorliegt, und dessen „totale“ (d. h. bis ins letzte Partikel reichende) Rekonstruktion. Dieser Vorgehensweise liegt die Auffassung zugrunde, dass alles im Text Enthaltene motiviert ist (im Sinne von: eine Bedeutung hat).

- Das Wörtlichkeitsprinzip und das Lesartenprinzip: Es dürfen nur solche Schlüsse gezogen werden, die vom Text erzwungen sind. Interpretationen im Stil „was sein kann" sind nicht zulässig, also Lesarten, die zwar mit dem Text kompatibel sind, aber nicht zwingend diesen Schluss zulassen. Es dürfen daher nur diejenigen Lesarten expliziert werden, die in der Ausdrucksgestalt noch konkret markiert und von ihr erzwungen sind.

- Das Prinzip der Gültigkeit bzw. der Authentizität: Aus der Beachtung des Prinzips der Gültigkeit bzw. Authentizität folgt, dass das „Authentische“ immer im Text markiert ist, wobei Authentizität nicht gleichbedeutend mit dem „Wahren“ oder einzig „Richtigen“ zu sehen ist - gerade das „Unwahre“ und „Fehlerhafte“ wird als authentisch und somit gültig betrachtet. Das Authentische ist das eigentliche, zu verifizierende Thema, die Primärbedeutung. Bei Texten kann eine Primärbedeutung von einer sekundären Ausdrucksform gerahmt werden, z. B. kann Ironie die sekundäre Rahmung einer Primärbedeutung sein. Diese Rahmung ist ebenfalls authentisch. Gemäß dem Prinzip der Wörtlichkeit muss sie, bei Annahme ihres Gebrauchs, im Text „markiert“, d.h. erkennbar sein. Die bloße Annahme von Rahmungen, ohne dass diese im Text markiert und von diesem erzwungen sind, ist für die Gegenstandserschließung hinderlich und nach der Vorgehensweise der Objektiven Hermeneutik nicht zulässig.

- Das Prinzip der Sequenzanalyse: Grundlegend für die Methode der Objektiven Hermeneutik ist die Sequenzialität, worunter kein triviales zeitliches und räumliches Nacheinander bzw. Hintereinander verstanden wird, sondern die mit jeder Einzelhandlung als Sequenzstelle sich vollziehende Öffnung neuer Optionen oder Schließung vorausgehend eröffneter Möglichkeiten. Es gibt ein 
Gesamt an Sequenzregeln, durch die an einer je gegebenen Sequenzstelle die sinnlogisch möglichen Anschlüsse erzeugt werden und auch die jeweils möglichen sinnlogisch kompatibel vorausgehenden Handlungen festgelegt sind und entsprechend erschlossen werden können.

\subsection{Schleuderschneeketten}

Der Film zeigt in einer Mischung aus Animation und realen Filmaufnahmen die Funktionsweise von Schleuderschneeketten. Bei plötzlich auftretendem Schnee lassen sich vom Fahrerhaus aus die Schneeketten unter die Antriebsräder zuschalten. Ein Schwenkarm, an dessen Ende sich ein drehbares Kettenrad befindet, fährt aus. Trifft das Kettenrad auf den Antriebsreifen, beginn es sich zu drehen und die Kettenstränge werden zwischen Lauffläche und Fahrbahn geschleudert und bringen sogenannten Grip unter die Antriebsräder.
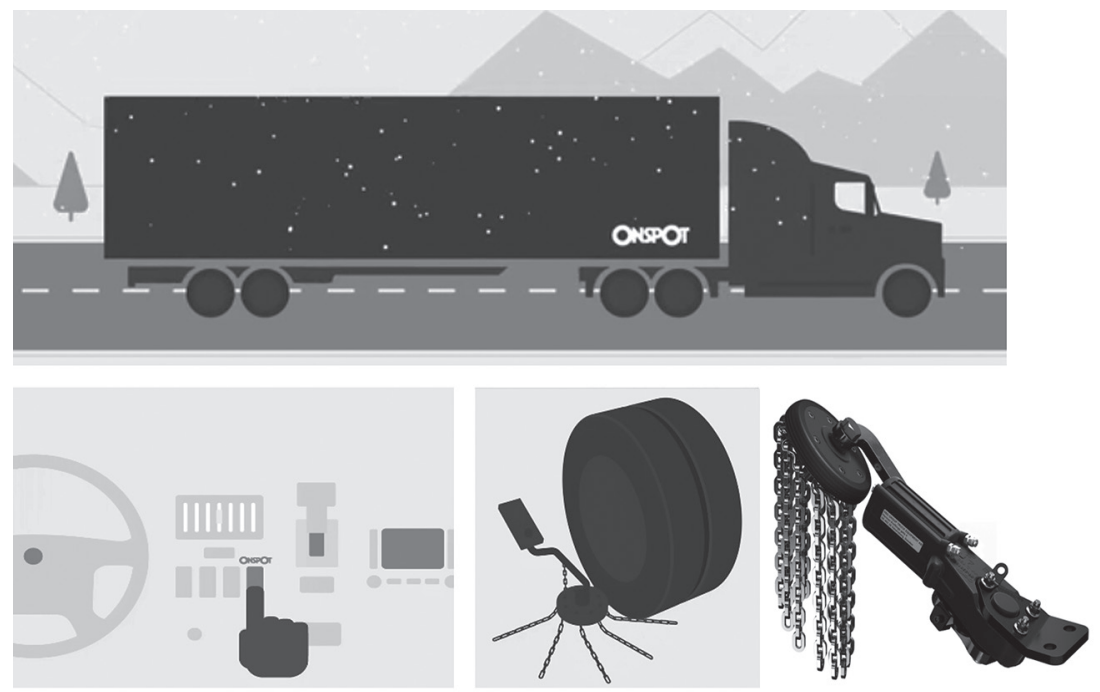

Abb. 1: Exemplarische Screenshots aus dem Technik-Stummfilm „Schleuderschneeketten“

Eine Gesprächssequenz stellt sich wie folgt dar:

A (Junge, 11 Jahre): Schleuderschneeketten steht da. Was auch immer Schleuderschneeketten sind.

B (Junge, 11 Jahre): Ein LKW. Und es schneit. Der schlingert.

A: Ah cool, Automatik. Oder was? Nein echt jetzt? Nice, der hat dann da so Ketten, echt jetzt, die dann den Schnee jetzt direkt unter den Rädern wegmachen? Nice, ist ja richtig nice. Alter, die schleudern dann da so rum. Das will ich nochmal sehen. Aber wie gehen die dann da wieder weg, weil die klemmen doch dann unter dem Rad fest? 
Ah nee, anscheinend nicht, ist vielleicht zu rutschig? Hey, aber cool. Als ob's das wirklich gibt. Das würde ich gerne mal sehen. Nice.

B: Damit kann man natürlich überall fahren. Coole Aufnahmen.

A: Alter, als ob es die tatsächlich gibt, ist ja richtig cool. Aber wie verstaut man die dann bitte, ich mein, die haben da unten doch gar keinen Platz? Naja. Da! Da klappen sie schon ein. Also die gehen dann einfach unter die Räder und dadurch ist eben kein Schnee mehr unter den Rädern, sondern diese Teile. Man hat aber nichts zu tun. Man muss nicht aussteigen, man muss keine Schneeketten dranmachen. Das sind halt einfach diese Teile. Ein Knopfdruck reicht. Cool.

In dieser Gesprächssequenz hat A den Dateinamen „Schleuderschneeketten“ gelesen. Sein Satz „Was auch immer Schleuderschneeketten sind“ "zeigt, dass er zunächst keinen Anknüpfungspunkt an diese Bezeichnung findet. B drückt zu den Themen „LKW“, „schneien“ und „schlingern“ kurz aber prägnant die im Film dargestellte Gesamtsituation aus - das Schneien entpuppt sich ja als wesentliches, nicht nur rahmendes Element des Films. "Schlingern“ ist dabei ein sehr treffender, bildlich-lebhafter Beschrieb für die Bewegung des LKW.

A benennt mit der Feststellung „Automatik" ein Schlüsselelement des Films, nämlich das Neue an der Erfindung: der Mensch muss nicht mehr aussteigen und die Schneeketten anlegen, sondern die technische Vorrichtung ermöglicht es ihm, die Schneeketten per Knopfdruck zu aktivieren. In den Äußerungen „echt jetzt“,

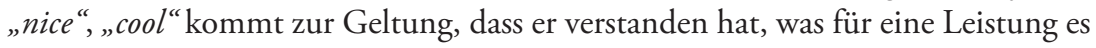
ist, sich so etwas auszudenken.

Die Vermutung von A besteht in der Vorstellung, die Ketten würden den Schnee unter den Rädern beiseiteschieben, wegräumen. Was ihn ausgehend von dieser Vermutung irritiert ist, dass die Ketten aber immer wieder durch das Rad eingeklemmt werden, und so am Wegschleudern von Schnee gehindert werden. Er kommt nicht auf die alternative Deutung, dass die Ketten quasi Griffigkeit herstellende Elemente sind.

Die Frage „Aber wie verstaut man die [Schneeketten] dann bitte, ich mein, die haben da unten doch gar keinen Platz?" weist darauf hin, dass A keine Vorstellung davon hat, wie viel Platz unter einem LKW vorhanden ist (auch „normale“ Schneeketten oder ganze Ersatzräder werden oft dort aufgehängt gelagert), dass ihm die Raumverhältnisse unter einem LKW nicht deutlich vor Augen stehen.

\subsection{Dübel}

Dargestellt ist eine Wand im Querschnitt, in die ein Loch gebohrt ist. In dieses wird ein Dübel gesteckt und anschließend eine Schraube eingedreht. Zu sehen ist, wie beim Eindrehen der Schraube der Dübel verformt wird und es wird darzustellen versucht, wie es zwischen Dübel und Wand zu einem Form- und Kraftschluss kommt. 
Eine Gesprächssequenz lautete wie folgt:

A (Junge, 11 Jahre): Das ist ein, eine Wand, mit einem Loch drin, und da ist ein Dübel und eine Schraube.

B (Junge, 11 Jahre): Und jetzt wird der Dübel in die Wand geschoben, und die Widerhaken hindern ihn daran, dass er wieder rausgeht. Die Schraube wird reingeschraubt. Und der Dübel geht aber nicht bis zum Ende vom Loch, sondern nur fast, und die Schraube berührt aber das Ende.

A: War es das schon? Hhm.

B: Nochmal schauen. Jetzt geht der Dübel in die Wand. Dann verhaken sich diese Häk-, Dinger.

A: Und warum schraubt man die Schraube nicht gleich in die Wand? Zu locker. Oder was?

B: Hhm.

A: $H h m$.

Diese Zweiergruppe kämpfte bei der Erschließung mit der Frage nach der Funktion des Dübels - die Funktion eines Dübels scheint eher außerhalb dessen zu liegen, was Kinder bereits erfahren haben. Junge A nimmt zunächst eine sehr sachliche Beschreibung der Situation vor, bewegt sich auf der konkret-logischen Ebene dessen, was man wahrnimmt. In seiner Beschreibung ist alles Wesentliche benannt. Das Wort „Dübel“ ist ihm vertraut. Junge B formuliert den Vorgang des Einsteckens des Dübels. Sein Ausdruck „den Dübel schieben“ trifft den Vorgang gut, auch wenn ein Techniker diesen Ausdruck eher nicht verwenden würde. Das Detail "Widerhaken" wird vom Kind erkannt - die Widerhaken sind technisch vor allem wichtig, wenn man einen Dübel in ein Loch in der Decke steckt, also über Kopf arbeitet. Auch die Funktion der Widerhaken ist erkannt worden die Verhinderung des Herausfallens bzw. Zurückrutschens. Nicht klar ist, ob der Junge das Hindern am Herausgehen in diesem Moment aus dem Filmkontext ableitet oder schon vorher Bescheid gewusst hat. Es ist möglich, dass er es im Film erschlossen hat, denn in der Animation ist gut zu erkennen, wie die Häkchen zusammengedrückt werden und sich dann im Lochinnern wieder abspreizen. Was in der Gesprächssequenz fehlt, ist das Erschließen oder Erkennen des Prinzips von Dübel und Schraube, also dass die Schraube den Dübel auseinanderpresst, so dass es zu einem Kraft- und Formschluss kommt - was den eigentlichen Grund darstellt, warum die Schraube dann in der Wand hält. Diese Funktion der Fixierung der Schraube durch den Dübel wurde nicht erkannt.

Was Junge B noch feststellt, ist, dass der Dübel etwas kürzer ist als das Loch. Tatsächlich müssen Schraube und Dübel nicht 100\%ig übereinpassen; wichtig ist nur, dass das Loch in Betonwänden so tief gebohrt ist, dass die Schraube nicht gegen die hintere Wand stößt (denn dann würde sie durch die Drehbewegung den Dübel wieder herausbefördern). Wäre der Dübel sehr viel länger als die Schraube, 
könnte das sich auch ungünstig auf die Haltekraft auswirken, weil er sich dann in die Länge verziehen kann.

Interessant ist, dass Junge B das Gefühl hat, etwas Wichtiges übersehen oder die Sache noch nicht ganz durchdrungen zu haben. Er macht Gebrauch von der Möglichkeit, einen Teil des Films noch einmal anzusehen. Junge A stellt dann mit der Warum-Frage die Frage nach der Funktion des Dübels. Die für die Funktion wichtige Bewegung des Dübels - das Auseinanderdrücken der zwei Dübelschalen - haben beide Jungen nicht gesehen bzw. beachtet; sie haben in Bezug auf den Dübel nur die Widerhakenbewegung wahrgenommen. Dübel werden verwendet in Wänden, in denen sich eine Schraube kein Gewinde schneiden kann - entweder, weil die Wand zu hart ist, oder weil die Wand Schaden nehmen könnte, z. B. weil sie sehr porös ist, oder weil die Wand Hohlräume hat (z. B. Gipsplattenwände - in diesen Wänden werden Spezialdübel eingesetzt, sog. Hohlraumdübel).

\section{Zusammenfassung des Erkenntnisgewinns}

Im Folgenden sollen Aussagen, zu denen die Analyse führt, zusammenfassend dargestellt werden. Der Erkenntnisgewinn wird der Übersicht halber in verschiedenen Themengruppen zusammengefasst.

\subsection{Bedeutung des medialen Zugangs „Technik-Stummfilme“ für Erschließungsprozesse}

Dass die rein mediale Präsentation der technischen Gegenstände bzw. Ereignisse ohne Möglichkeit eines handelnden Zugangs und der originalen Begegnung - an das kindliche Interesse anzuschließen und die Kinder zu motivieren vermochte, zeigte sich darin, dass fast alle Zweiergruppen entweder in ihrem Aufschrieb oder im Gesprächsverlauf Fragen formulierten.

Zudem zeigen die Gesprächsaufzeichnungen, dass sich die Kinder sehr konzentriert (d.h. die Kinder ließen sich kaum ablenken, die Erkenntnisgewinnung stand im Mittelpunkt ihrer Bemühungen) eine ganze Schulstunde lang mit den Stationen auseinandersetzten. Die Gespräche waren auf die Themen fokussiert; nach Abschluss einer Station suchten die Kinder unmittelbar die nächste Station auf und ließen sich auf das dort gezeigte Thema ein. Zum einen liegt hier der Erklärungsansatz nahe, dass die Kinder zeitlich länger anhaltenden Medienkonsum gewöhnt sind und dass der Reiz von Medien auf Kinder stark ist. Ein weiterer Erklärungsansatz gründet sich auf der Tatsache, dass bei den Stummfilmen die Darstellungsweise „Animation“ dominierte und dass diese Darstellungsweise auf Kinder eine Faszination ausübt, weil die Dinge einerseits realistisch wirken, andererseits Blicke ins Innere ermöglichen oder Prozesse des Auseinander- und Zusam- 
menbaus in Form animierter Explosionszeichnungen verfolgt werden können. Es lässt sich zudem die Vermutung aufstellen, dass die Stationen, an denen von auffallend vielen Kindern Fragen formuliert waren, Themen zum Gegenstand hatten, die an die Lebenswelt der Kinder anschließen und Kinder so genügend Anknüpfungspunkte haben, um Fragen zu stellen. Ebenfalls fiel auf, dass die Kinder von sich aus die Möglichkeit nutzen, die Filme zwischendurch anzuhalten oder sich Passagen wiederholt anzuschauen.

\subsection{Bedeutung der Anknüpfung an die kindliche Lebenswelt für Erschließungsprozesse}

Für die Filme, die bei vielen Kindern auf großes Interesse stießen, gilt in besonderem Maße, dass sie unmittelbar an die Lebenswelt der Kinder anschließen („Reißverschluss“, „Handbohrmaschine“). Die Äußerungen und Aufschriebe der Kinder bezüglich des Films „Schleuderschneeketten“ zeigen, dass die Kinder an dem Gegenstand und Mechanismus interessiert waren, obwohl dieser keinen engen Bezug zur kindlichen Lebenswelt aufweist - die Vermutung ist, dass er den Kindern wegen seines hohen Grads an Konkretheit und wegen der guten Wahrnehmbarkeit aller Abläufe erfassbar erschien. Dass Filme schwer zu verstehen sind, äußerten Kinder in Bezug auf die Filme „Wagenheber“, „Handschwengelpumpe“, „Kugelschreiber“, „Mähdrescher" und „Fahrradschaltung“. Kompliziert macht das Erschließen dieser Filme, dass nicht alles, was passiert, sichtbar ist. Nicht sichtbar sind im Fall der Handschwengelpumpe z. B. die sog- und druckbasierten Vorgänge oder beim Wagenheber der Gewindezugmechanismus.

\subsection{Bedeutung der Sprache und des Dialogs für Erschließungsprozesse}

Sowohl in den Aufschrieben als auch in den Gesprächssequenzen zeigte sich, dass die Kinder überwiegend von Beobachtungen und der Versprachlichung der Beobachtung ausgehen. Diesen Schritt lassen sie nicht aus, obwohl denkbar wäre, dass er nach dem Motto: das haben wir gesehen, das müssen wir nicht nochmals erwähnen, weggelassen würde. Das Gegenteil ist der Fall - die Kinder teilen sich gegenseitig mit, was sie gesehen haben. Oftmals begannen die Sequenzen damit, dass die Kinder die Situation im Film beschrieben. Im Prozess des Erschließens teilten die Kinder sich immer wieder ihre bisherigen Erfahrungen und ihr Vorwissen mit. Den Kindern ist der Austausch darüber, woher sie etwas kennen, erkennbar wichtig; im Dialog regen sie sich gegenseitig an, Erinnerungen, Erfahrungen und Anknüpfungspunkte gegenseitig zu aktivieren.

Die Kinder kamen in ihren Gesprächen ohne exakte Bezeichnungen, elaborierte Formulierungen und ohne komplexe sprachliche Strukturen (wenn-dann, je-desto, weil, damit) aus. Häufig fanden die Kinder von selbst sehr treffende Bezeichnungen für Gegenstände, Bauteile oder Vorgänge (vgl. z. B. die Bezeich- 
nung „Hebelarm“ für den Pumpenarm der Handschwengelpumpe oder das Verb „schlingern“ zur Beschreibung des LKW-Fahrverhaltens in Schnee, Kap. 4.1). In manchen Äußerungen zeigt sich auch, dass es den Kindern möglich ist, sich an gezeigte, ihnen unbekannte Elemente mit eigenen Wortschöpfungen anzunähern (z. B. im Fall des Wagenhebers „Drehaufwagen“, „Autolüpfer“). Ungenauigkeiten oder das Fehlen von Begriffen sowie Auslassungen oder Platzfüller (z. B. ,irgendwie") weisen darauf hin, dass bestimmte Vorstellungen noch nicht gebildet wurden. Für Dinge, die einer sich bildenden Person noch unklar sind oder zu denen kein Bezug da ist, fehlen ihr oft die Worte. Gleichzeitig darf nicht aus dem Blick geraten, dass trotz solcher Verständnislücken oft auch viel Wesentliches erfasst wird.

\subsection{Bedeutung des rekonstruktiven Erschließens für technische Bildungsprozesse}

Bezüglich des rekonstruktiven Schließens fällt auf, dass die Kinder sich besonders häufig des abduktiven Schließens in Form des Analogieschlusses bedienten (Hinweise darauf liefern Formulierungen wie „das sieht aus wie“, „das ist wie bei“, „das kenne ich von“, „das gibt es auch bei“). Die Kinder machen Gebrauch vom konkret-logischen Denken. Dies zeigt sich u. a. darin, dass sie sich auf die unmittelbar vorliegenden, beobachtbaren Merkmale eines Gegenstands beziehen und die räumliche Orientierung bei Beschreibungen eine große Rolle spielt, z. B. in Form einer Innen-Außen-, Oben-Unten-, Rechts-Links Strukturierung von Beschreibungen und dass Beschreibungen generell wichtig sind und ständig von den Kindern vorgenommen werden. Überwiegend verfügten die Kinder nicht über fertige Konzepte; am häufigsten trat ansatzweise Konzeptwissen bezüglich des Hebelgesetzes auf (ein Beispiel an der Station „Wagenheber“: „Das hat irgendwas mit Hebelkraft zu tun, also das muss ja eigentlich ganz leicht sein, man kann ja nicht einfach mit Kraft ein Auto hochstemmen").

In Bezug auf die Kinder fiel auf, dass einige in der Lage waren, das Wesentliche in den Filmen zu erkennen und zu benennen und die gezeigten Prozesse zu ordnen und zu strukturieren sowie Einzelheiten wegzulassen und Kompliziertes auf Einfaches zu überführen. Aber nicht immer waren die Kinder in der Lage, das Wesentliche an einem Gegenstand oder Vorgang zu erfassen; manchmal blieb die Erschließung eher oberflächlich. Dies ist ein Hinweis darauf, dass es notwendig ist, zusätzlich zum Stummfilm den Gegenstand oder das Ereignis direkt vor sich zu haben und sich handelnd-erkundend damit auseinanderzusetzen sowie ggf. mit Hilfe eines sokratisch-mäeutischen Gesprächs auf wichtige Fragen oder Merkmale aufmerksam zu werden.

Beim Aufspüren von Zusammenhängen machten die Kinder am ehesten Gebrauch von der Logik der Funktionsweise (vgl. Wagenschein 1923/1996, 10, Kap. 2.4) in der Art, dass sie sich entlang der ablaufenden Handlung vorwärtshangeln 
(ein Beispiel an der Station „Heißklebepistole“: „Da ist jetzt der Kleber drin. Der wird dann, wenn man drückt, immer weiter nach vorne geschoben, und da vorne wird er erhitzt, und dann kommt er eben flüssig vorne raus, aber hinten ist er fest. ". Neben Fragen nach der Funktion traten „Was ist das?-Fragen“ auf, mit Hilfe derer Kinder sich nach der Bezeichnung erkundigen (phonologische Elaboration). Dass die Kinder anfangen, über Bedeutungen nachzudenken, ist erkennbar an Fragen wie "Was kann man damit machen?", "Wozu braucht man das?", "Warum hat es ein Gewinde?", „Wie ist die Bewegung?" (semantische Elaboration).

\subsection{Der Erkenntnisgewinn im Kontext des aktuellen Forschungsstands}

Bezüglich der Bedeutung des medialen Zugangs für Erschließungsprozesse decken sich die Befunde der vorliegenden Studie mit eigenen vorangegangenen Forschungsergebnissen, u. a. mit dem Forschungsergebnis, dass Fragen der Kinder ein wichtiger Indikator für ihre Neugier sind (vgl. Schumann 2018a) und dass Filme die originale Begegnung in Bildungsprozessen nicht ersetzen können (vgl. Schumann 2015). Dass Zugänge qua Medien ein Potential für Erschließungsprozesse haben, andererseits auch Risiken bergen, ist bisher zu wenig genauer untersucht worden. In der vorliegenden Studie zeigte sich, dass Medien einerseits fokussierend wirken und viele Möglichkeiten für eine gute Beobachtung in sich bergen, andererseits aber auch eine sehr oberflächliche Rezeption der Inhalte erfolgen kann. Über die Wirkung von verschiedenen Filmformaten auf Kinder, z. B. die festgestellte Beliebtheit von Animationen bei Kindern, ist bisher ebenfalls kaum etwas bekannt.

Die Bedeutung der Anknüpfung von Inhalten an die kindliche Lebenswelt für Erschließungsprozesse ist hingegen vielfach untersucht (vgl. u. a. GDSU 2013, Hempel 2007). Gut in der vorliegenden Studie erkennbar war, dass Kinder auch dann ihnen eher fremde Gegenstände oder Vorgänge zu erschließen vermochten, wenn sich diese auf der Basis von sinnlicher Wahrnehmung im Modus konkret-logischen Denkens als gut erschließbar erwiesen, auch wenn Vorwissen und Vorerfahrungen fehlten.

Wie eingangs dargestellt, wird eine nähere Untersuchung der Bedeutung der Sprache und des Dialogs für Erschließungsprozesse gefordert. Bezüglich der Versprachlichung von Beobachtungen und der Auswahl von Wörtern im Rahmen von Beschreibungen stimmen die Befunde mit der Annahme Wagenscheins überein, Kinder könnten selbst gut feststellen, wann ein Begriff besonders treffend oder klärend wirke und kämen in Erschließungsprozessen auch ohne Fachbegriffe aus (Wagenschein 1923/1996). In den Dialogen fiel vor allem die gemeinsame Fokussierung auf einen Sachverhalt auf und das den Dialogen innewohnende Potential, dass Kinder erfahren können, wie andere denselben Sachverhalt wahrnehmen und interpretieren, dass es einen Unterschied gibt zwischen „meinen“ und „wissen“ und dass es übergeordnete Probleme und Teilprobleme gibt. Die Kinder 
scheuten sich nicht, sich Momente des Nicht-Verstehens einzugestehen und auch mit Vermutungen, derer sie sich nicht sicher waren, zu den Dialogen beizutragen. Köhnlein weist bezüglich solcher Dialoge darauf hin, dass gelingende Kommunikation das „Wagnis, Eigenes in die Unwägbarkeiten des Diskurses einzubringen“ bedeute und dass es notwendig sei, „die Perspektive der anderen produktiv aufzunehmen, mitzuvollziehen, was andere denken, und zu versuchen, den Gesamtzusammenhang zu überblicken" (Köhnlein 2012, 122f.).

Über die Bedeutung des rekonstruktiven Erschließens ist, vor allem im Zusammenhang mit Überlegungen zum Konstruktivismus, bereits viel nachgedacht worden, gleichzeitig wird in den Schulen überwiegend das deduktive Vorgehen praktiziert. Über das in der vorliegenden Studie häufiger anzutreffende abduktive Erschließen von Kindern, z. B. mit Hilfe von Analogieschlüssen, gibt es nur wenige Untersuchungen (Hoffmann o.J.). Hinweise auf das Bedürfnis von Kindern, im konkret-logischen Modus erschließen zu können, welche die vorliegende Studie enthält, ließen sich bereits in vorangegangenen Studien finden (Geiss \& Schumann 2015). Die Frage, ob Kinder von sich aus in Erschließungsprozessen in der Lage sind, Wesentliches zu identifizieren und wann es Bedarf an partieller Unterstützung gibt, ist in der sachunterrichtsdidaktischen Forschung vor allem Thema in den vielfältigen Auseinandersetzungen unter den Stichwörtern „Instruktion“ und „Konstruktion“ (vgl. Giest, Heran-Dörr \& Archie 2012). In Bezug auf sich auf technische Inhalte beziehende Erschließungsprozesse ließ sich in der vorliegenden Studie feststellen, dass für die Kinder häufig die Fragen nach der Funktion bzw. des Funktionierens im Vordergrund standen - laut Wagenschein ist dies ein sinnvoller und möglicher Weg der Erschließens von Technik (Wagenschein 1923/1996, 10).

\section{Ableitungen bezüglich Möglichkeiten der Unterstützung rekonstruktiver Erschließungsprozesse}

Die zweite Forschungsfrage lautete: Lässt sich aus den Erkenntnissen ableiten, wie rekonstruktive Erschließungsprozesse, bezogen auf technische Ereignisse, zusätzlich zu dem Angebot „Stummfilme“, unterstützt werden können? Im Folgenden werden die wichtigsten diesbezüglich generierten Thesen dargestellt.

\subsection{Die Unterstützung rekonstruktiver Erschließungsprozesse durch Materialien und die Ermöglichung eines handlungsorientierten Zugangs}

Auch wenn die Kinder erstaunlich ausdauernd mit Hilfe des medialen Zugangs versuchten, Gegenstände und Abläufe zu durchdringen, griffen sie an den Stationen mit zur Verfügung gestelltem Material überwiegend sofort nach diesem. 
Von der originalen Begegnung (Copei 1955, Roth 1970) scheint für Kinder der Primarstufe ein großer Reiz auszugehen. Manche Fragen lassen sich auch nur auf der Basis der gemachten Erfahrungen stellen. Einige Prinzipien und Funktionen allerdings lassen sich weder im Film noch im Original, sondern fast nur im Modell sichtbar machen. Modelle sollten, wenn sie im Technikunterricht auf der Primarstufe eingesetzt werden, von ihrer Beschaffenheit her so wenig abstrahiert und so nah am Original wie möglich sein, damit die Prinzipien so eindeutig wie möglich zur Wirkung kommen.

\subsection{Die Unterstützung durch Film und Bilddarstellungen}

Sowohl bei der originalen Begegnung als auch bei der Begegnung im Film kann in Bezug auf die Erschließung ein dialektisches Wechselspiel aus Beobachtung von Markierungen am Gegenstand und das Bilden von Lesarten erlebt werden. Teilweise sind Film-Protokolle sogar besonders wertvoll, denn der Film kann beispielsweise angehalten und der Moment damit eingefroren werden, der Film kann verlangsamt abgespielt werden oder man hat bei animierten Filmen die Möglichkeit, auf einfache Weise und sofort Einblicke ins Innere zu erhalten. Insgesamt gilt: Die Güte des zugrundeliegenden Protokolls spielt für das Bilden von Lesarten und das Generieren von Deutungsvorschlägen eine zentrale Rolle. Wenn ein Film prägnant ein Phänomen oder einen technischen Vorgang wiedergibt und Details gut erkennbar sind, die Proportionen stimmen etc., unterstützt dies den Erkenntnisgewinnungsprozess maßgeblich.

In der Studie griffen die Kinder von sich aus auf die Möglichkeit zurück, von Zeichnungen Gebrauch zu machen, um einen Sachverhalt in ihren Aufschrieben darzustellen. Im Fall älterer Schülerinnen und Schüler der Primarstufe lassen sich durch Zeichnungen Prinzipien bzw. Mechanismen klären, die sich evtl. der Wahrnehmung stärker entziehen, z. B. der Aufbau und die Funktion des Bohrfutters der Handbohrmaschine oder der Sitz der Kugel im Kugelschreiber. Die Zeichnungen der Kinder ermöglichen auch eine (Eigen-)Diagnose ihres Verständnisses. Eine Handbohrmaschine aus dem Gedächtnis zeichnen kann der Annahme nach beispielsweise nur diejenige Person, die die Bauteile und ihr Zusammenspiel verstanden und bewusst wahrgenommen hat.

\subsection{Die Unterstützung durch Texte}

Eine bildungswirksame Herausforderung für Kinder könnte es sein, selbst Kommentierungen zu einem Film zu entwerfen. Kinder können beim Verfassen von Erklärtexten lernen, auf bestimmte Kriterien zu achten, z. B. auf Einfachheit (leicht verständliche Texte, anschauliche Texte), Übersichtlichkeit/Geordnetheit (leicht nachzuvollziehende Gliederung), Kürze (Prägnanz) und Anregungsintensität. Die Kinder könnten sich für die von ihnen erstellten Redetexte gegenseitig 
Feedback geben, also Vorschläge kritisch in Hinblick auf die Verständlichkeit von Texten für Laien anschauen.

Nachdenken lässt sich auch über die Möglichkeit, Sich-Bildenden zusätzlich zu technischen Gegenständen, Materialien und Modellen erklärende Sachtexte anzubieten. Fachtexte weisen neben dem Fachwortschatz eine hohe inhaltliche Dichte auf und stellen gehobene Anforderungen an Leserinnen und Leser, u. a. weil sie viele Nebensatz- und Passivkonstruktionen enthalten (Senn 2008). Ein anderer Ansatz, von Texten Gebrauch zu machen, mit dem man rekonstruktives Erschließen eher unterstützt, könnte darin bestehen, dass die Kinder eigene Textvorschläge entwerfen - in der vorliegenden Studie haben sie das bereits freiwillig getan, darin also offenbar einen Sinn oder Mehrwert gesehen.

\subsection{Die Unterstützung durch den sokratisch-mäeutischen Dialog und Impulsfragen}

Es fiel auf, dass in den Fällen, in denen Kinder beim Erschließen kaum über die Beobachtung und Beschreibung hinauskamen, von ihnen häufig wichtige Details übersehen wurden oder ihnen für tiefergehende Erschließungsprozesse wichtige Fragen fehlten. Fragen sind zentral in ihrer Funktion, einen Anker zu setzen, von dem aus man einen Gegenstand weiterführend beobachten und untersuchen kann. Ein Beispiel: Bei der rekonstruktiven Erkundung eines Kugelschreibers kann wichtige Erkenntnis generiert werden, wenn der Frage nachgegangen wird, warum ein Kugelschreiber Kugelschreiber heißt. Die Frage kann eine Suche und Auseinandersetzung mit der Kugel auslösen und zu weiteren Fragen führen, z. B. zu der Frage, wie es kommt, dass die Kugel weder aus dem Schreibgerät herausfällt noch ins Innere rutscht oder zu der Frage, woran es liegen kann, wenn ein Kugelschreiber nicht schreibt (z. B. ist die Tinte leer oder die Kugel eingetrocknet oder das Papier so glatt, dass es für die Mine keinen ausreichenden Widerstand leistet, um rollen zu können). Fragen fördern ein vertieftes Erschließen, schützen vor Oberflächlichkeit und lenken den Blick auf für Technikkonzepte entscheidend wichtige Details.

\section{Fazit}

Vor dem Hintergrund des Anspruchs des Sachunterrichts, an die Rekonstruktionsleistungen von Kindern anschließen und Kinder beim Versuch, sich die technische Welt zu erschließen, unterstützen zu wollen, scheint es notwendig zu sein, Erschließungsprozesse von Kindern in Bezug auf Technik näher zu untersuchen. In der vorliegenden Studie zeigt sich, dass Kinder in der Primarstufe interessiert daran sind, sich rekonstruktiv erschließend mit technischen Ereignissen bzw. Ge- 
genständen auseinanderzusetzen, und dass sie eine hohe Anstrengungsbereitschaft mitbringen, wenn es darum geht, Funktionsweisen und Bauweisen zu erkunden. Kinder sind aber, so zeigte die Studie, nicht immer in der Lage, selbstständig und nur auf der Basis der eingesetzten Stummfilme und des Peergroup-Dialogs auf wesentliche Prinzipien und die entsprechend entscheidenden, technischen Details aufmerksam zu werden. Die Studie gibt Hinweise darauf, dass es hilfreich sein kann, Kinder auf bestimmte Bauteile oder Funktionsmechanismen aufmerksam zu machen, z. B. durch Impulsfragen (z. B. „Warum heißt der Kugelschreiber Kugelschreiber?") oder mittels sokratisch-mäeutischer Dialoge.

Die Unterstützung rekonstruktiver Erschließungsprozesse von Kindern birgt die Chance, dass die Kinder über die Ebene des Beobachtens und der Beschreibung hinauskommen und die Erfahrung machen können, wie man rekonstruktiv erschließend zu Interpretationen über die Welt gelangen kann. Die Erschließung von sowohl einzelnen Bauteilen als auch das Erfassen der Bauteil-Beziehungen zueinander kann dem Potential nach zu einer, den Dingen einen Sinn gebenden, Erschließung von Technikkonzepten führen.

\section{Literatur}

Aeschlimann, U.; Buck, P.; Hugel, A.; Ostergaard, E.; Rehm, M. \& Rittersbacker, C. (2008): Phänomenologische Naturwissenschaftsdidaktik. In: GDCP (Hrsg.): Kompetenzen, Kompetenzmodelle, Kompetenzentwicklung. Tagungsband der Jahrestagung 2007. Berlin: LIT Verlag, 179-181.

Apolin, M. (2004): Sprache im Physikunterricht. In: PLUS LUCIS 1/2004, 15-18.

Archie, C. (2016): Sachunterricht sprachsensibel gestalten. In: Landesinstitut für Schule und Medien Berlin-Brandenburg (Hrsg.): Lernarrangements für den Sachunterricht, Teil 1, 20-28.

Bielmeier, B. (2012): Wissensarbeiter sind die Zukunft. In: www.fortschrittsforum.de, August 2012. Online unter: http://www.fortschrittsforum.de/debattieren/bildung-modernisierung/artikel/article/wissensarbeiter-sind-die-zukunft-1/page.pdf (Abrufdatum: 21.09.2018).

Butler, J. (2006): Haß spricht: Zur Politik des Performativen. Frankfurt/Main: Suhrkamp.

Copei, F. (1955): Der fruchtbare Moment im Bildungsprozeß. 3. Auflage 1955. Heidelberg: Quelle \& Meyer.

Dewey, J. (1933): How we think. A restatement of the relation of reflective thinking to the education process. Lexington, Massachusetts: D. C. Heath and Company.

Donaldson, M. (1991): Wie Kinder denken. Intelligenz und Schulversagen. München, Zürich: Piper.

Einsiedler, W. (2015): Geschichte der Grundschulpädagogik. Entwicklungen in Westdeutschland und in der DDR. Heilbrunn: Kinkhardt.

Franz, U.; Giest, H.; Hartinger, A.; Heinrich-Dönges, A. \& Reinhoffer, B. (2018): Editorial. In: U. Franz, H. Giest, A. Hartinger, A. Heinrich-Dönges \& B. Reinhoffer (Hrsg.): Handeln im Sachunterricht. GDSU, Band 28. Heilbrunn: Klinkhardt, 9-16.

Geiss, R. \& Schumann, S. (2014): Erschließungsprozesse im Sachunterricht - Ansprüche, Konzepte, Praxis oder: Wie kann Unterricht die Entwicklung eines Forscherhabitus unterstützen? In: www. widerstreit-sachunterricht.de, Nr. 20, April 2014.

Geiss, R. \& Schumann, S. (2015): Orientierung als ein Anspruch des Sachunterrichts. Wie kann Sachunterricht in der Grundschule Kindern zu Orientierung verhelfen? www.widerstreit-sachunter- 


\section{Svantje Schumann}

richt.de, Beiheft Nr. 21, 2015. Online unter: https://www2.hu-berlin.de/wsu/ebeneI/superworte/ zumsach/schu_gei.pdf (Abrufdatum: 01.10.2018).

Gervé, F. \& Mayer, J. (2018): Handlungsorientierung in Doppeldeckern: Forschungsbasierte Seminarentwicklung und Demokratielernen im Planspiel In: Jahresband 28, 191-198.

Gesellschaft für Didaktik des Sachunterrichts (GDSU) (2013): Perspektivrahmen Sachunterricht. Bad Heilbrunn: Klinkhardt.

Giest, H. (2004): Handlungsorientiertes Lernen. In: D. Pech \& A. Kaiser (Hrsg.): Neuere Konzeptionen und Zielsetzungen im Sachunterricht. Basiswissen Sachunterricht Band 2. Baltmannsweiler: Schneider, 90-98.

Giest, H.; Heran-Dörr, E. \& Archie, C. (Hrsg.) (2012): Lernen und Lehren im Sachunterricht. Zum Verhältnis von Konstruktion und Instruktion. GDSU Band 22. Bad Heilbrunn: Klinkhardt.

Goedhart, M. (1999): Das Gespräch als Mittel zur Begriffsentwicklung, erläutert am Beispiel von Siedepunktbegriffen. In: GDCP (Hrsg.): Zur Didaktik der Physik und Chemie. Probleme und Perspektiven. Tagungsband der Jahrestagung 1998. Alsbach, Bergstraße: Leuchtturm-Verlag, 97-99.

Gottwald, A. (2012/2016): Sprachförderndes Experimentieren im Sachunterricht. Wiesbaden: Springer VS. Hartinger, A. (1997): Interessenförderung: eine Studie zum Sachunterricht. Bad Heilbrunn: Klinkhardt.

Hartinger, A. (1997): Interessenförderung: eine Studie zum Sachunterricht. Bad Heilbrunn: Klinkhardt.

Heck, U.; Weber, C. \& Baumgartner, M. (2009): Lernen in Erfahrungsräumen. Baltmannsweiler: Schneider.

Hempel, M. (2007): Diagnostik der kindlichen Lebenswelt als Voraussetzung zur Förderung des Kompetenzerwerbs der Lernenden. In: R. Lauterbach, A. Hartinger, B. Feige \& D. Cech (Hrsg.): Kompetenzerwerb im Sachunterricht fördern und erfassen. GDSU Band 17. Bad Heilbrunn: Klinkhardt.

Hoffmann, M. H. G. (o.J.): Lernende lernen abduktiv: eine Methodologie kreativen Denkens. Online unter: http://www.prism.gatech.edu/ - mh327/03-MH-abduktiv-Lernen.pdf (Abrufdatum: $01.10 .2018)$.

Kahlert, J. (2009): Der Sachunterricht und seine Didaktik. 3. Auflage. Bad Heilbrunn: Klinkhardt.

Kaiser, A. (2004). Praxisbuch handelnder Sachunterricht. Band 2. Baltmannsweiler: Schneider.

Köhnlein, W. (2008/2011): Die Bildungsaufgaben des Sachunterrichts und der genetische Zugriff auf die Welt. Vortrag, gehalten 2008 an der Universität Gifu, Japan. In: GDSU-Journal März 2011, Heft 1. Online unter: http://www.gdsu.de/gdsu/wp-content/uploads/2011/02/koehnlein_1_11_a. pdf (Abrufdatum: 22.06.2018).

Köhnlein, W. (2012): Sachunterricht und Bildung. Bad Heilbrunn: Klinkhardt.

Kruse, S. \& Labudde, P. (2016): Technology Education in Switzerland. In: M. J. de Vries, S. Fletcher, S. Kruse, P. Labudde, M. Lang, I. Mammes, C. Max, D. Münk, B. Nicholl, J. Strobel \& M. Winterbottom (Hrsg.): Technology Education Today: International Perspectives. Münster, New York: Waxmann, 59-74.

Leber, M. \& Oevermann, U. (1994): Möglichkeiten der Therapieverlaufsanalyse in der objektiven Hermeneutik. Eine exemplarische Analyse der ersten Minuten einer Fokaltherapie aus der Ulmer Textbank „Der Student“. In: D. Garz \& K. Kraimer (Hrsg.): Die Welt als Text. Theorie, Kritik und Praxis der objektiven Hermeneutik. Frankfurt am Main: Suhrkamp, 383-427.

Linke, S. (2013): Aktuelle fachdidaktische Grundlagen und Entwicklungsperspektiven im Erkenntniskomplex Technik. In: H.-J. Fischer, H. Giest \& D. Pech (Hrsg.): Der Sachunterricht und seine Didaktik. Bestände prüfen und Perspektiven entwickeln. GDSU Band 23. Bad Heilbrunn: Klinkhardt.

Merzyn, G. (1998): Sprache im naturwissenschaftlichen Unterricht. In: Praxis der Naturwissenschaften, Heft 2/47, 1998, 203-205 und 243-246. 
Merzyn, G. (2008): Naturwissenschaften, Mathematik, Technik - immer unbeliebter? Die Konkurrenz von Schulfächern um das Interesse der Jugend im Spiegel vielfältiger Untersuchungen. Baltmannsweiler: Schneider.

Mikelskis-Seifert, S. \& Gromadecki, U. (2006): Naturwissenschaftliche Arbeitsweisen. Unterricht zur Einführung in die naturwissenschaftlichen Denk- und Arbeitsweisen. In: Naturwissenschaften im Unterricht Physik. Heft 93, Juni 2006, 17. Jahrgang, 31-37.

Neugebauer, N. \& Nodari, C. (2017): Förderung der Schulsprache in allen Fächern: Praxisvorschläge für Schulen in einem mehrsprachigen Umfeld. 5. korrigierte Auflage. Bern: Schulbuchverlag plus.

Oevermann, U.; Allert, T.; Konau, E. \& Krambeck, J. (1979): Die Methodologie einer „objektiven Hermeneutik" und ihre allgemeine forschungslogische Bedeutung in den Sozialwissenschaften. In: Soeffner, H. G. (Hrsg.): Interpretative Verfahren in den Sozial- und Textwissenschaften. Stuttgart: J. B. Metzlersche Verlagsbuchhandlung, 352-434.

Oevermann, U. (1996): Krise und Muße. Struktureigenschaften ästhetischer Erfahrung aus soziologischer Sicht. Vortrag am 19.6. in der Städel Schule, Frankfurt am Main. Online unter: http:// www.agoh.de/cms/de/downloads/uebersicht/oeffentlich/oevermann/Oevermann-Ulrich-Krise-und-Mu\%C3\%9Fe-Struktur-eigenschaften-\%C3\%A4sthetischer-Erfahrung-aus-soziologischer-Sicht-\%281996\%29/ (Abrufdatum: 04.09.2018).

Ostergaard, E. \& Hugo, A. (2008): Vom Phänomen zum Begriff - und zurück. Entwicklung phänomenologischer Unterrichtskompetenzen. In: GDCP (Hrsg.): Kompetenzen, Kompetenzmodelle, Kompetenzentwicklung. Tagungsband der Jahrestagung 2007. Berlin: LIT Verlag, 182-184.

Roth, H. (1970): Pädagogische Psychologie des Lehrens und Lernens. 12. Auflage 1970. Hannover: Schroedel.

Royar, T. \& Streit, C. (2010): MATHElino. Kinder begleiten auf mathematischen Entdeckungsreisen. Seelze: Kallmeyer.

Sachs, B. (2001): Technikunterricht - Bedingungen und Perspektiven. In: tu - Zeitschrift für Technik im Unterricht, Heft 100, 5-12.

Schönknecht, G. \& Maier, P. (2012): Diagnose und Förderung im Sachunterricht. Kiel: IPN.

Schumann, S. (2015): Der Wert der originalen Begegnung. «Die wichtigste Voraussetzung für Bildung ist die $\mathrm{Mu}$ ße». Interview: Jennifer Degen. Zoomagazin Basel, 10-11. Online unter: http://www. zoobasel.ch/cgi-bin/magazin/files/Die_wichtigste_Voraussetzung_ist_die_ Musse.pdf (Abrufdatum: 10.09.2018).

Schumann, S. (2018a): Naturwissenschaftsdidaktik in der Frühpädagogik. Die Bedeutung der sozialen Kooperation für die frühe naturwissenschaftliche Bildung. Aachen: Shaker Verlag. Zugl. Habilitationsschrift Universität Bremen.

Schumann, S. (2018b): Technik-Stummfilme. Online-Plattform für die Aus- und Weiterbildung von PädagogInnen, die Nutzung in Schulen und weitere Forschung. Online unter: http://web.fhnw.ch/ ph/projekte/technik-stummfilme (Abrufdatum: 10.07.2018).

Senn, S. (2008): Sachtexte lesen - in allen Fächern. In: Rundschreiben Zentrum Lesen - Pädagogische Hochschule der FHNW - Institut Forschung und Entwicklung 14/2008.

Soostmeyer, M. (1998): Zur Sache Sachunterricht. Begründung eines situations-, handlungs- und sachorientierten Unterrichts in der Grundschule. Frankfurt am Main: Lang.

Soostmeyer, M. (2002): Genetischer Sachunterricht - Unterrichtsbeispiele und Unterrichtsanalysen zum naturwissenschaftlichen Denken bei Kindern in konstruktivistischer Sicht. Baltmannsweiler: Schneider.

Tomcin, R. \& Reiners, C. S. (2009): Auf malerischem Weg zur Chemie. Zum didaktischen Potential von Chemie-Foto-Stroys. In: Chemkon 16/1, 6-13.

Wagenschein, M. (1968): Die Sprache im Physikunterricht. In: Zeitschrift für Pädagogik, 7. Beiheft. Weinheim, Berlin, Basel, 125-142. 


\section{6 | Svantje Schumann}

Wagenschein, M. (1976): Die pädagogische Dimension der Physik. Braunschweig: Georg Westermann Verlag.

Wagenschein, M. (1999): Verstehen lehren. Genetisch - Sokratisch - Exemplarisch. Weinheim und Basel: Beltz.

Wagenschein, M. (1923/1996): Über die Förderung der sprachlichen Ausdrucksfähigkeit durch den mathematischen und naturwissenschaftlichen Unterricht. Hausarbeit für die Staatsprüfung für das höhere Lehramt. April 1996 herausgegeben vom Martin-Wagenschein-Archiv, Hasliberg Goldern.

Wagenschein, M. (2005): Was bleibt? Verfolgt am Beispiel der Physik. In: www.widerstreit-sachunterricht.de, Nr. 5, Oktober 2005.

Wernet, A. (2006): Hermeneutik - Kasuistik - Fallverstehen. Eine Einführung. Stuttgart: Kohlhammer Verlag. 